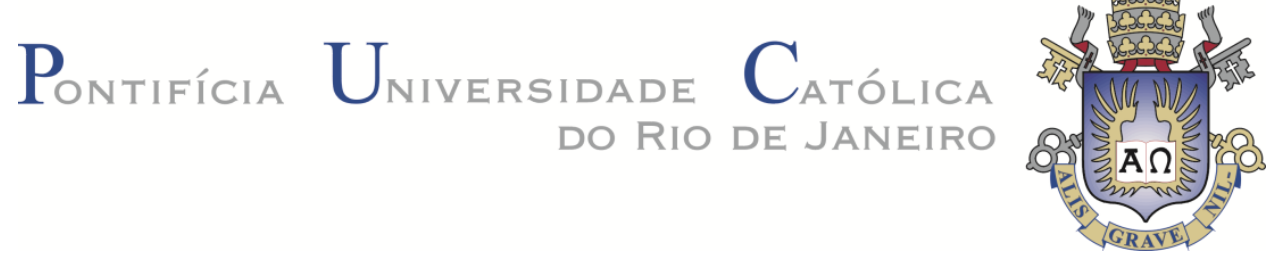

Reinaldo Bellini Gonçalves

Modelagem Teórica e Computacional de Dendrímeros para o Transporte de Tuberculostáticos

Tese apresentada ao Programa de Pós-graduação em Engenharia Elétrica da PUC-Rio como requisito parcial para obtenção do título de Doutor em Engenharia Elétrica.

Orientador: Prof. Marco Aurélio C. Pacheco Co-Orientador: Prof. Ricardo Bicca de Alencastro Co-Orientador: Prof. Vanessa L. Rodrigues Furtado 


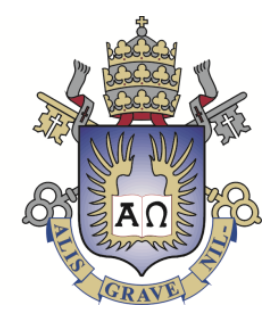

Reinaldo Bellini Gonçalves

\title{
Modelagem Teórica e Computacional de Dendrímeros para o Transporte de Tuberculostáticos
}

Tese apresentada como requisito parcial para obtenção do grau de doutor pelo Programa de Pós Graduação em Engenharia Elétrica do Departamento de Engenharia Elétrica do Centro Técnico Científico da PUC-Rio. Aprovada pela Comissão Examinadora abaixo assinada.

\author{
Prof. Marco Aurélio C. Pacheco \\ Orientador \\ Departamento de Engenharia Elétrica -PUC-RIO \\ Prof. Ricardo Bicca de Alencastro \\ Co-orientador \\ Instituto de Química -UFRJ \\ Prof.Vanessa L. Rodrigues Furtado \\ Co-orientador \\ Instituto de Química -UFRJ \\ Prof. Ernesto Raúl Caffarena \\ PROCC -FIOCRUZ
}

Prof. Omar Paranaíba Vilela Neto DCC -UFMG

Prof. Juan Lazo Lazo

ICA -PUC-RIO

Prof. Bruno Araújo Cautiero Horta Laboratory of Computational Chemistry Biochemistry EPF -LAUSANNE

Prof. José Eugênio leal Coordenador setorial do centro Técnico Científico- PUC/RIO

Rio de Janeiro, 28 de junho de 2012 
Todos os direitos reservados. É proibida a reprodução total ou parcial do trabalho sem a autorização da universidade, do autor e do orientador.

\section{Reinaldo Bellini Gonçalves}

Graduou-se em Ciências Exatas pelo Centro de Ensino Superior de Juiz de Fora com Licenciatura Plena em Matemática. Mestrado em Modelagem Computacional pelo Laboratório Nacional de Computação Científica (LNCC). Atuando em Matemática Aplicada na Área de Modelagem Computacional.

Ficha Catalográfica

Gonçalves, Reinaldo Bellini

Modelagem teórica e computacional de dendrímeros para o transporte de tuberculostáticos / Reinaldo Bellini Gonçalves ; orientador: Marco Aurélio C. Pacheco ; co-orientador: Ricardo Bicca de Alencastro ; co-orientador: Vanessa L. Rodrigues Furtado. - 2012.

152 f. : il. (color.) ; $30 \mathrm{~cm}$

Tese (doutorado)-Pontifícia Universidade Católica do Rio de Janeiro, Departamento de Engenharia Elétrica, 2012.

Inclui bibliografia

CDD: 621.3 


\section{Agradecimentos}

A CAPES, PUC/Rio e o ICA PUC/Rio pela ajuda financeira e material de pesquisa sem os quais o trabalho não poderia ter sido realizado.

Mãe vencemos de novo. Quantas vezes choramos, quantos anos ficamos separados por este objetivo, mas enfim coisas de Deus. Aquele que nos leva por caminhos inesperados, desconhecidos, árduos, mas que são planos que a nossa pequena compreensão perante o seu infinito poder não podemos compreender. Estranho né, aquele menino que nasceu e ficava dentro da caixa de papelão da mercearia, que todos achavam que não ia dar em nada. Mas enfim eles não contavam com o meu maior trunfo VOCÊ. Mãe obrigado por sempre ser tudo em minha vida, sem você nada seria possível. Obrigado por me apoiar qualquer que sejam as circunstâncias que a vida me coloca. Que Deus seja louvado por ter me dado você como mãe.

Ao meu pai que me ensinou que quando as coisas estão ruins é melhor trabalhar mais, levantar mais cedo, orar e no fim tudo acaba bem. Desistir nunca, vencer sempre. Ao meu pai que me ensinou a ter honra e caráter o que me tornou um homem de virtudes.

Ao meu irmão que sempre me apoiou nessa incrível jornada, que nos separa em alguns instantes da vida, mas que nos torna mais fortes a cada encontro. Quanto aperto passamos por causa de alguns fatos inexplicáveis da vida, mas que superamos a cada dia, a cada instante, certos que no final venceremos todos. Esteja certo de uma coisa que eu sempre tive o impossível não existe.

A dona Júlia que há muito tempo faz parte da nossa vida, e que sempre vibra com nossas vitórias neste estranho mundo de desencontros.

A quem briga, chora, ri, sofre, a quem me aguenta em qualquer situação. A quem faz tody a noite, a quem sabe um pouquinho desta vitória, a quem divide as alegrias e tristezas. A quem sempre soube o que eu já era antes de ser. E o que é mais importante, a quem é fiel, companheira e não se vai à primeira batalha a ser vencida nem a abandona quando esta é árdua demais. A você Márcia.

A professora Vanessa furtado, por ter sido paciente todos estes anos. Por me mostrar a beleza dos dendrímeros. Por ser alguém que devo me espelhar, que esta sempre disposta a trabalhar. Se preciso, viramos a noite trabalhando, mas 
impossível, aprendi com a senhora mais uma vez que nada é impossível. Muito obrigado por ter me dado a honra de ter sido aluno de tão nobre pessoa.

Ao professor Marco Aurelio que quando estou desmotivado, basta ir em sua sala e sair com algumas idéias para conquistar o mundo. Um dos caras mais inteligentes que eu tive o prazer de conhecer, que sempre propõe o impossível pois o possível não tem graça. Ao cara que mesmo em dificuldades fora da tese sempre me deu apoio. Ao meu grande orientador que sou muito grato por ter confiado às aulas, alunos de IC, palestras e apresentações. Obrigado por ter me feito crescer tanto nestes últimos anos.

Ao professor Ricardo Bicca, que é um gigante da química, ou melhor, um gigante da ciência. Obrigado por estar sempre atento durante a tese, por ter me aceito de braços abertos em seu grupo e por me motivar a cada encontro. Sinto-me muito honrado por ter sido orientado por um gigante da ciência como o senhor.

Ao Ernesto, por ser um cara de profundos conhecimentos, por ter me ajudado quando mais precisei, por ter me acalmado nos momentos de desespero da tese. Por apesar de não ter conseguido trabalhar com ele ter me acompanhado desde o mestrado. Por ser um cara não só de grandes conhecimentos, mas de grande caráter, de grande simplicidade, o que mostra mais ainda sua infinita sabedoria. Acho que já tive muitas vidas e com certeza muitos mestres e você um dos principais. Muito obrigado.

Ao Laurent que me preparou para enfrentar um exército, que me tornou auto didata, que me preparou para qualquer situação. Que apesar de longe ainda sabe o que ando fazendo. Que tenho muito orgulho de ter sido seu aluno. Que é um dos grandes gênios da ciência.

A professora Judith Felcman (in memorian) por ter sido paciente, por ter me apoiado, por ter me ensinado química, e o mais importante por ter me mostrado que devo fazer tudo com muito amor e detreminação.

Ao doutor Alberto H. Haad, um dos maiores matemáticos que já conheci, que me mostrou que eu era capaz. Obrigado, até quando te encontrava pela rua era suficiente para me motivar, só pelo prazer de te cumprimentar, meu velho mestre. Muito orgulho de ser seu discípulo e por carregar por onde passo seu nome com muita honra e glória.

A Sônia Eunice Maciel por ser minha motivadora, por ter confiado em mim quando mais precisei. Minha mestra quanta saudade daqueles velhos tempos, mas 
você já sabia que seu aluno ia longe, obrigado e desculpe aquele choro, mas a verdade é que ainda choro por lembrar de vocês meus grandes mestres.

Aos meus amigos do lab, Iury, pelas conversas, motivações e ajudas. Ao Alan pela amizade e companheirismo. As minhas novas amigas Jacqueline e Nataly e minha velha companheira de jornada Lú. Ao Júnior pela amizade de sempre. Ao Omar por ter sido meu amigo, ter me confiado o grupo de nano, por ter me ajudado muito quando precisei. Muito obrigado.

A quem escolheu seguir outros caminhos, outras histórias, outros aprendizados. A você que me acompanhou três anos durante esta luta incansável, obrigado.

"Se cheguei até aqui é porque estava apoiado sobre ombros de gigantes"

Sônia, Alberto, Laurent, Ernesto, Marco Aurelio, Vanessa, Ricardo Bicca, Judith.

Muitas vidas, muitos mestres... 


\section{Resumo}

Gonçalves, Reinaldo Bellini; Pacheco, Marco Aurélio Cavalcante; Alencastro, Ricardo Bicca; Furtado, Vanessa Rodrigues. Modelagem Teórica e Computacional de Dendrímeros para o Transporte de Tuberculostáticos. Rio de Janeiro, 2012. 152p. Tese de Doutorado Departamento de Engenharia Elétrica, Pontifícia Universidade Católica do Rio de Janeiro.

Neste trabalho inicialmente foram modeladas as estruturas do dendrímero PAMAM G4 em diferentes estados de protonação utilizando-se o programa Hyperchem. A seguir essas estruturas foram inseridas em uma caixa d'água e simuladas por dinâmicamolecular, tendo sido observado um aumento de volume do dendrímero à medida que o grau de protonação foi aumentado, com a formação de uma estrutura mais aberta comparativamente à do dendrímero não protonado. Uma vez conhecidas as topologias do dendrímero PAMAM em diferentes $\mathrm{pH}$, foram incluídas moléculas de rifampicina nas cavidades existentes no interior do dendrímero em $\mathrm{pH}$ neutro simulado, através de um algoritmo desenvolvido com a finalidade de otimizar este encapsulamento, o que definiu como número máximo de 20 moléculas de rifampicina complexadas no interior do dendrímero e resultou em uma redução importante no custo computacional. Após estes acoplamentos pelo algoritmo desenvolvido, o complexo foi simulado através de dinâmica molecular tendo sido verificado que se mantinha estável em $\mathrm{pH}$ neutro ao longo do tempo.O estudo da possível liberação das moléculas de rifampicina do complexo rifampicina/PAMAM G4 por dinâmica molecular, mostrou que as moléculas de rifampicina são liberadas gradativamente em $\mathrm{pH}$ ácido. Os resultados computacionais foram validados por resultados experimentais obtidos em um trabalho desenvolvido em colaboração entre 0 IQ/UFRJ e o IPEC/FIOCRUZ, cujo objetivo foi caracterizar e determinar a atividade tuberculostática de complexos rifampicina/PAMAM G4.

\section{Palavras-chave}

Dinâmica molecular; Dendrimeros; Algoritmos; Nanotecnologia; Tuberculose. 


\section{Abstract}

Gonçalves, Reinaldo Bellini; Pacheco, Marco Aurélio Cavalcante (Advisor); Alencastro, Ricardo Bicca; Furtado, Vanessa Rodrigues. Theoretical and Computational Modeling of Dendrimers for Drug Delivery. Rio de Janeiro, 2012. 152p. PhD Thesis Departamento de Engenharia Elétrica, Pontifícia Universidade Católica do Rio de Janeiro.

The structures of PAMAM G4 dendrimer in different protonation states were initially modeled using the program Hyperchem. After this procedure the structures were inserted in a box of water and simulated with molecular dynamics. Was observed an increase in volume of the dendrimer when degree of protonation was increased. Once known the topologies of PAMAM dendrimer at different $\mathrm{pH}$, the rifampin was included in cavities of dendrimer in Neutral and low $\mathrm{pH}$. Using an algorithm designed for the purpose of optimizing this encapsulation, were encountered 20 molecules inside the cavities of dendrimer. This procedure resulted in a low computational cost for molecular dynamics because the molecules were in a optimal position in the structure of dendrimer. After these couplings of the systems these were simulated with molecular dynamics for observation of capacity in transport of dendrimer these molecules in neutral $\mathrm{pH}$ and liberation in low $\mathrm{pH}$. The study of the possible release of the complex molecules of rifampicin (rifampicin / PAMAM G4) showed that the molecules are released gradually of the structures at low $\mathrm{pH}$. The computational results were validated by experimental results on the work developed in collaboration between IQ / UFRJ and IPEC / FIOCRUZ, whose objective was to characterize and determine the tuberculostatic activity of complex.

\section{Keywords}

Molecular dynamics; Dendrimers; Algorithms; Nanotechnology; Tuberculosis. 


\section{Sumário}

1. Introdução 18

1.1. Motivação 18

1.2. Objetivos do Trabalho 19

1.3. Desenvolvimento do Trabalho 19

1.4. Organização da Tese 20

2. Transporte de Fármacos 22

2.1. Dendrímeros 22

2.1.1. Carreadores de Medicamentos 22

2.1.2. Dendrímeros 23

2.1.3. Dendrímeros PAMAM 25

2.1.4. Matemática do Dendrímero PAMAM 27

2.1.5. Propriedades Estruturais 28

2.1.6. Dendrímeros como Nanocarreadores 31

2.1.7. Interações entre Dendrímeros e Drogas 32

2.1.8. Toxicidade 33

2.1.9. Solubilidade 34

2.1.10. Interações com Membranas 34

2.1.11. Entrada do Dendrímero na Célula 35

2.1.12. Simulação Computacional dos Dendrímeros 36

2.2. Rifampicina e Tuberculose 38

2.2.1. Introdução à Tuberculose 38

2.2.2. Genoma do Mycobacterium Tuberculosis 39

2.2.3. Patologia 40

2.2.4. Sintomas 42

2.2.5. Resistência do Bacilo as Drogas 42

2.2.6. Introdução aos fármacos 42

2.2.7. Vias de Administração e Excreção 43

2.2.8. Membrana Celular 43

2.2.9. Tratamento da Tuberculose 45 
2.2.10. Rifampicina 46

2.2.12. Resistência a Rifampicina 47

3. Complexo - O Modelo Prático onde o trabalho se baseia. 48

4. Dinâmica Molecular 54

4.1. Simulação Computacional 54

4.2. Etapas da Simulação por Dinâmica Molecular 55

4.3. Configurações Iniciais dos Sistemas 55

4.4. Inserção do Solvente no Soluto 55

4.5. Condições Periódicas de Contorno 58

4.6. Campo de Força 58

4.7. Otimização de Geometria 60

4.8. Simulação Temporal dos Sistemas $\quad 61$

4.9. Velocidades Iniciais 62

4.10. Integração Numérica da equação de Movimento 62

4.11. Acoplamento de Temperatura 63

4.12. Acoplamento de Pressão 64

4.13. Análises de Propriedades Estruturais 65

5. Metodologia Utilizada no Trabalho 68

5.1. Modelagem das Moléculas 68

5.1.1. Dendrímeros 68

5.1.2. Rifampicina $\quad 70$

5.2. Dinâmica Molecular dos Dendrímeros 71

5.3. Campo de Força Utilizado 72

5.4. Carga dos Dendrímeros e Rifampicina 73

5.5. Inserção do Sistema em Solvente 73

5.6. Otimização 74

5.7. Dinâmica Molecular dos Dendrímeros $\quad 75$

5.8. Medidas das Propriedades $\quad 76$

5.9. Construção do Algoritmo de Acoplamento 76

5.9.1. Algoritmo 1 
5.10. Modelagem por Dinâmica Molecular dos Complexos Formados em Diferentes $\mathrm{pH}$.

6. Resultados e Discussão 85

6.1. Otimização 85

6.2. Simulações no Vácuo

6.2.1. Energia Cinética 86

6.2.2. Energia Total do Sistema 86

6.2.3. Raio de Giro 87

6.2.4. Distribuição Radial $\quad 87$

6.2.5. Distribuição Espacial 89

6.3. Simulação com Solvente Explícito, Fase de equilíbrio 89

6.3.1. Energia Total do sistema no Equilíbrio 90

6.3.2. Energia Cinética do sistema no Equilíbrio 91

6.3.3. Análise do RMSD na Fase de Equilíbrio 92

6.4. Simulação com Solvente Explícito, Fase de Produção 94

6.4.1. Energia Total dos Sistemas (Solvente Explícito) 94

6.4.2. Energia Cinética dos Sistemas (Solvente Explícito) 96

6.4.3. Raio de Giro 97

6.4.4. Análise de ĺons na Estrutura 99

6.4.5. Distribuição Radial de Moléculas de Água 100

6.4.8. Distância Média das Aminas da Periferia do Dendrímero ao Centro. 105

6.4.9. Análise do Volume das estruturas 105

6.4.10. Ligações de Hidrogênio Intermoleculares 106

6.4.11. Ligações de Hidrogênio Intramoleculares 107

6.4.12. Distribuição das Aminas de Superfície. 108

6.4.13. Superfície Acessível ao Solvente 109

6.5. Algoritmo de acoplamento na estrutura dos dendrímeros $\quad 110$

6.5.1. Busca com raio de $0,5 \mathrm{~nm}$ do centro 110

6.5.2. Busca com raio de $0,1 \mathrm{~nm}$ do centro 111

6.5.4. Análise de Energia para a inserção de 20 moléculas 113 
6.5.5. Algoritmo de Acoplamento com tratamento de colisões atômicas

6.6. Simulações de Complexos Rifampicina-Dendrímero

6.6.1. Complexo de Inclusão

120

6.6.2. Análise de RMSD

121

6.6.3. Energia Cinética e Total do Sistema na

121

6.6.4. Fase de Produção

122

6.6.6. Energia Cinética e Total do Sistema

123

6.6.7. Raio de Giro 124

6.6.8. Número de Moléculas de Água Interiores ao Dendrímero

125

6.6.9. Mudanças Estruturais das Moléculas de Rifampicina

127

6.6.10. Distribuição Radial

128

6.6.11. Análise de ligações de hidrogênio

130

6.6.12. Deslocamento Médio das Moléculas no Complexo

6.7. Liberação das moléculas de Rifampicina (Dendrimero em pH Ácido).

6.7.2. Energia Cinética e Total do Sistema (Fase de Equilíbrio) 135

6.7.3. Análise de RMSD (Fase de Equilíbrio) 136

6.7.4. Raio de Giro (Fase de Equilíbrio) 137

6.7.5. Liberação Sustentada (Fase de Equilíbrio) 137

6.7.7. Energia Cinética e Total do Sistema (Fase de Produção) 139

6.7.8. RMSD(Fase de Produção) 140

6.7.9. Continuação da Liberação Sustentada (Fase de Produção) 141

7. Conclusões

8. Referências 


\section{Lista de figuras}

Figura 1: Representação esquemática das partes de um dendrímero (núcleo, interior e superfície).

Figura 2: Representação esquemática da estrutura do dendrímero.

Figura 3: Representação de dendrímeros com diferentes características.

Figura 4: Dendrímero de Geração 1.

Figura 5: Representação de um dendrímero PAMAM de geração 10.26

Figura 6: Reações sucessivas da formação do dendrímero PAMAM. 28

Figura 7: Dendrimero PAMAM de geração 4.

Figura 8: Propriedades estruturais dos dendrímeros PAMAM. 30

Figura 9: Bacilo de Koch. 40

Figura 10: Esquema de uma membrana celular. 44

Figura 11: Molécula de Rifampicina. 46

Figura 12: Espectros no IV. $\quad 49$

Figura 13: Espectro de infravermelho do hidrogênio. 50

Figura 14: Crescimento do M. tuberculosisH37Rv ATCC 27294.

Figura 15: Liberação da rifampicina do complexo. 53

Figura 16: Caixa de simulação. 56

Figura 17: Modelo de água do tipo SPC. 56

Figura 18: Modelos de água TIP4P e TIP5P. 57

Figura 19: Caixa de simulação. $\quad 58$

Figura 20: Distribuição radial em torno de uma partícula. 66

Figura 21: Superfície acessível ao solvente. 67

Figura 22: Dendrímeros PAMAM geração 1 a 4.

Figura 23: Molécula de Rifampicina.

Figura 24: Caixa de Simulação Utilizada. $\quad 74$

Figura 25: Rotações da cadeia lateral da rifampicina. $\quad 80$

Figura 26: Moléculas sendo geradas em todo o espaço de busca. 82

Figura 27: Moléculas geradas somente nos pontos de ótimos. 83

Figura 28: Energia Cinética do sistema no vácuo. 86

Figura 29: Energia total do sistema no vácuo. 86 
Figura 30: Raio de giro do sistema no vácuo.

Figura 31: Distribuição radial em torno do centro de massa do dendrímero.

Figura 32: Ligações de hidrogênio entre aminas primárias e carbonila. 88

Figura 33: Distribuição espacial do dendrímero.

Figura 34: Energia total do sistema, para o pH básico.

Figura 35: Energia total do sistema em pH neutro. 90

Figura 36: Energia total do sistema para o pH acido. 91

Figura 37: Energia Cinética do sistema para o pH básico. 91

Figura 38: Energia Cinética do sistema para o pH neutro. 92

Figura 39: Energia Cinética (pH ácido). 92

Figura 40: RMSD contra a primeira estrutura da dinâmica, pH básico. 93

Figura 41: RMSD contra a primeira estrutura da dinâmica, $\mathrm{pH}$ neutro 93

Figura 42: RMSD contra a primeira estrutura da dinâmica, $\mathrm{pH}$ ácido. 94

Figura 43: Energia Total para o pH básico 95

Figura 44: Energia total para o pH neutro 95

Figura 45: Energia total para o pH neutro. 95

Figura 46: Energia cinética para o pH básico 96

Figura 47: Energia cinética para o pH neutro. 96

Figura 48: Energia cinética para o pH ácido. $\quad 97$

Figura 49: Raio de giro para o pH básico 97

Figura 50: Raio de giro para o pH neutro. 98

Figura 51: Raio de giro para o pH ácido 98

Figura 52: Função radial de átomos das moléculas de água. 100

Figura 53: Distribuição radial do dendrímero. 101

Figura 54: Estruturas do dendrímero PAMAM no vácuo. 102

Figura 55: Estruturas do dendrímero PAMAM no vácuo. 103

Figura 56: Estruturas do dendrímero PAMAM no vácuo. 104

Figura 57: Distribuição radial em torno do centro de massa do dendrímero. 108

Figura 58: Superfície acessível ao solvente 109

Figura 59: Gráfico de energia potencial das conformações 1, 2 e $3 . \quad 113$

Figura 60: Estrutura mais estável encontrada pelo algoritmo. 117

Figura 61: Estrutura média do complexo de inclusão. 120 
Figura 62: RMSD na fase de equilíbrio do complexo de Inclusão.

Figura 63: Gráfico de energia cinética. 122

Figura 64: Energia total do sistema simulado. 122

Figura 65: RMSD durante a trajetória entre rifampicinas e dendrímero. 123

Figura 66: Energia cinética do sistema na fase de produção. 124

Figura 67: Energia total do sistema. 124

Figura 68: Raio de giro do dendrímero. 125

Figura 69: RMSD. 128

Figura 70: Distribuição radial do complexo de inclusão. 129

Figura 71: Estruturas de liberação do complexo. 135

Figura 72: Energia cinética do sistema. 135

Figura 73: Energia total do sistema. 136

Figura 74: Gráfico de RMSD das moléculas de dendrímero 136

Figura 75: Energia cinética Fase de produção. 139

Figura 76: Energia total do sistema. 140

Figura 77: RMSD das moléculas de rifampicina em relação ao dendrímero. 140

Figura 78: Complexo de inclusão com 20 moléculas de rifampicina. 142

Figura 79: Liberação sustentada das moléculas de rifampicina. 142 


\section{Lista de Tabelas}

Tabela 1: Tabela representativa das propriedades do dendrímero PAMAM.

Tabela 2: Drogas complexadas ou conjugadas com o dendrímero PAMAM.

Tabela 4: Estruturas dos dendrímeros PAMAM.

Tabela 5: Número de átomos e cargas por geração do dendrímero G0 até G4.

Tabela 6: Número de átomos e cargas por geração do dendrímero G0 até G4.

Tabela 7: Número de átomos e cargas por geração do dendrímero G0 até G4.

Tabela 8: Tabela do custo computacional das simulações. $\quad 72$

Tabela 9: Propriedades dos sistemas a serem simulados. $\quad 74$

Tabela 10: Tempo total de simulação dos sistemas. 77

Tabela 11: Tempos de dinâmicas moleculares comparadas. 78

Tabela 12: Minimização de energia com o algoritmo Steep-Descent. $\quad 85$

Tabela 13: Minimização de energia com o algoritmo Conjugated Gradient.

Tabela 14: Raio de giro em nanômetros. 87

Tabela 15: Raio de giro. $\quad 98$

Tabela 16: Número de íons inseridos no dendrímero. 99

Tabela 17: Distância média das aminas da periferia ao centro do dendrímero em nanômetros. $\quad 105$

Tabela 18: Volume dos dendrímeros em nanômetros 106

Tabela 19: Número de ligações de hidrogênio encontradas durante a dinâmica molecular nos oito picos segundos finais. 106

Tabela 20: Número de ligações de hidrogênio encontradas durante a dinâmica molecular nos oito picos segundos finais. 107

Tabela 21: Tabela da área acessível ao solvente, em nm2. 109 
Tabela 22: Análise com raio de no máximo 5 angstroms do centro do dendrímero.

Tabela 23: Análise com raio de no máximo 10 angstroms do centro. 112

Tabela 24: Análise com raio de no máximo 10 angstroms do centro. 113

Tabela 25: Algoritmo com inserção aleatória de distâncias comparado ao algoritmo que insere moléculas sem estas colisões. 115

Tabela 26: Taxa de sucesso para o algoritmo sem colisões atômicas. 115

Tabela 27: Algoritmo com distribuição de Cauchy comparado ao algoritmo onde as moléculas eram distribuídas aleatoriamente.

Tabela 28: Distribuições estatísticas utilizadas no trabalho.

Tabela 29: Ligações de hidrogênio, com dendrímero complexado com rifampicina e sem estas moléculas.

Tabela 30: RMSD entre moléculas de rifampicina.

Tabela 31: Tabela que apresenta os resultados das ligações de hidrogênio no 10 ns finais de simulação.

Tabela 32: Distância média em nanômetros entre moléculas e o centro de massa do dendrímero.

Tabela 33: Raio de giro comparando dendrímero com moléculas inseridas e sem estas.

Tabela 34: Distância média das moléculas de rifampicina ao centro de massa do dendrímero.

Tabela 35: Ligações de hidrogênio médias comparadas.

Tabela 36:Liberação das moléculas de rifampicina do complexo. 